\title{
Transcranial direct current stimulation for depression: 3-week, randomised, sham-controlled trial ${ }^{\dagger}$
}

Colleen K. Loo, Angelo Alonzo, Donel Martin, Philip B. Mitchell, Veronica Galvez and Perminder Sachdev

\section{Background}

Preliminary evidence suggests transcranial direct current stimulation (tDCS) has antidepressant efficacy.

\section{Aims \\ To further investigate the efficacy of tDCS in a double-blind sham-controlled trial (registered at www.clinicaltrials.gov: NCT00763230) \\ Method \\ Sixty-four participants with current depression received active or sham anodal tDCS to the left prefrontal cortex (2 mA, 15 sessions over 3 weeks), followed by a 3-week open-label active treatment phase. Mood and neuropsychological effects were assessed.}

\section{Results}

There was significantly greater improvement in mood after active than after sham treatment $(P<0.05)$, although no difference in responder rates (13\% in both groups). Attention and working memory improved after a single session of active but not sham tDCS $(P<0.05)$. There was no decline in neuropsychological functioning after 3-6 weeks of active stimulation. One participant with bipolar disorder became hypomanic after active tDCS.

\section{Conclusions}

Findings confirm earlier reports of the antidepressant efficacy and safety of tDCS. Vigilance for mood switching is advised when administering tDCS to individuals with bipolar disorder.

\section{Declaration of interest}

None.
Although the use of weak electrical currents to stimulate the brain has been described for centuries in the history of medicine, it has been reintroduced with higher intensity currents since 2000 as 'transcranial direct current stimulation' (tDCS). ${ }^{1}$ It involves passing a weak, depolarising current through the brain. This shifts the resting membrane potential, with anodal stimulation depolarising the soma of pyramidal cells, whereas cathodal stimulation results in hyperpolarisation. ${ }^{2}$ The effects of tDCS on neuronal excitability have now been demonstrated in numerous neuroimaging and physiological studies, providing a sound neurobiological basis for its use for neuromodulation in patient populations. ${ }^{1,3}$

Increased understanding of the effects of tDCS has been accompanied by application in clinical trials exploring its therapeutic potential in neurology and, more recently, psychiatry. ${ }^{1}$ Its potential role in depression has generated particular interest. Prior to 2000, early stimulation trials used relatively low current intensities $(0.02-0.5 \mathrm{~mA})$ with considerable variability in stimulation technique. As a result, outcomes were highly variable. ${ }^{3,4}$ Since then, the development of commercial equipment enabling the reliable delivery of currents in the 1-2 $\mathrm{mA}$ range has led to renewed interest in tDCS in clinical research. Since 2000, three sham-controlled trials investigating the efficacy of tDCS for treating depression have reported encouraging results. Fregni et $\mathrm{al}^{5}$ ( $n=10$, tDCS given at $1 \mathrm{~mA}, 20 \mathrm{~min}$ per session, 5 sessions on alternate days) and Boggio et al ${ }^{6}(n=40,2 \mathrm{~mA}, 20 \mathrm{~min}, 10$ sessions on consecutive weekdays) both found tDCS more effective than a sham control. In Loo et $a l^{7}(n=40,1 \mathrm{~mA}$, $20 \mathrm{~min}, 5$ sessions on alternate days followed by 5 further active treatments) clinically meaningful improvement was seen with active tDCS over 10 sessions of treatment, but differences

'See pp. 10-11, this issue. failed to reach statistical significance over the initial 5-session sham-controlled comparison period.

This present study further tested the efficacy of tDCS in individuals with current depression, administering stimulation at $2 \mathrm{~mA}$, for $20 \mathrm{~min}$ in 15 daily sessions, with a further 15 sessions on an open-label basis (registered at www.clinicaltrials.gov: NCT00763230). Acute and cumulative neuropsychological effects were also assessed with a detailed battery of tests chosen to specifically assess verbal memory and executive function.

\section{Method}

\section{Participants}

The study was approved by the human research ethics committee of the University of New South Wales and was conducted at the Black Dog Research Institute in Sydney. Recruitment began in September 2008 and the final 1-month follow-up was conducted in February 2011. The trial was closed after the intended sample size was reached (including an additional two participants who had already been screened as suitable and consented at the time of the decision to close the study). The sample size was estimated from the results of Fregni et al, ${ }^{5}$ the only published study of tDCS given in the 1-2 mA range in February 2007, and our pilot data in nine participants. From changes in responses to the Hamilton Rating Scale for Depression reported in Fregni et al ${ }^{5}$ (60\% mean reduction in the active group, $10 \%$ mean reduction in the sham group), assuming a baseline depression score of 20 (with a standard deviation of 7.5), a sample size of 10 participants per group (a total of 20 for the study) would have been required to detect an effect of the same magnitude with $80 \%$ power and $\alpha=0.05$. Our pilot data from the first nine participants of the study described in Loo et $\mathrm{al}^{7}$ showed a 4.5 -point difference in 
depression scores between active $(n=4)$ and sham $(n=5)$ tDCS treatment over a 1.5-week sham-controlled study period, with tDCS given at $1 \mathrm{~mA}$ for $20 \mathrm{~min}$. As the present study involved tDCS at $2 \mathrm{~mA}$ on consecutive weekdays, with sham and active treatment compared over a 15-session, 3-week study period, we estimated there would be at least a six-point difference between active and sham groups in depression scores at the end of the sham-controlled treatment phase. Using end of treatment mean scores of 17.3 (active group) and 23.3 (sham group), and a s.d. $=8.2$, based on our pilot data, the power analysis indicated that 31 participants per group (total sample 62 ) would be required to detect this effect with $80 \%$ power and $\alpha=0.05$.

Sixty-four participants with a DSM-IV ${ }^{8}$ major depressive episode and with a score of $\geqslant 20$ on the Montgomery-Åsberg Depression Rating Scale (MADRS) ${ }^{9}$ gave informed written consent and were enrolled as out-patients. Diagnosis was based on a structured assessment using the Mini-International Neuropsychiatric Interview $(\mathrm{MINI})^{10}$ and confirmed in a clinical interview by a study psychiatrist (C.K.L.). Exclusion criteria were other Axis I disorders, alcohol misuse, drug dependence or misuse, neurological disorders, electronic or metal implants, history of heart disease, neurological disorders, failure to respond to electroconvulsive therapy in the current depressive episode, pregnancy and concurrent treatment with medications that have been shown to alter effects of tDCS (benzodiazepines, anticonvulsants, dextromethorphan and pseudoephedrine). ${ }^{11,12}$ Treatment resistance was assessed for the current episode of depression as the number of failed adequate courses of antidepressant medications, and also according to the Maudsley Staging system. ${ }^{13}$

During the study, participants were either medication free or remained on antidepressant medications (to which they had failed to respond after an adequate treatment trial). For clinical and ethical reasons, participants were not required to withdraw from these medications if they or their treating clinicians had concerns about possible deterioration as a consequence of medication withdrawal. Any concurrent antidepressant medications were continued at stable doses, which had not been altered for at least 4 weeks prior to study entry.

\section{Study design}

Participants were stratified by gender and age and randomly assigned by a computer-generated random sequence to active $(n=33)$ or sham $(n=31)$ treatment over a 3 -week masked treatment phase. After being screened and signing consent, participants were enrolled and allocated to treatment groups by research staff who were not involved in mood ratings. The treatment assignment was indicated by a code on study treatment sheets, which were concealed from raters. Active or sham tDCS was administered every weekday (15 treatments in total), with participants and raters masked to group allocation. All participants were then offered an additional 3 weeks of open-label active tDCS, also administered every weekday. Participants who met the criterion for response (50\% improvement in MADRS score from baseline) were eligible to receive further sessions of tDCS on a weekly basis, over the 1-month follow-up period. After both the sham-controlled and open-label phases, participants were asked to guess their group allocation in the sham-controlled phase to assess integrity of the masking. Participants and raters were unmasked after the 6-week trial duration or at trial exit for participants who withdrew before trial completion.

\section{Treatment with tDCS}

The tDCS was administered by an Eldith DC-stimulator (NeuroConn GmbH, Germany) with the anode over the left dorsolateral prefrontal cortex, identified as $\mathrm{pF} 3$ on the international 10/20 EEG (electroencephalogram) system, and the cathode placed over the lateral aspect of the contralateral orbit, at the F8 position (10/20 system). Conductive rubber electrodes $\left(7 \times 5=35 \mathrm{~cm}^{2}\right)$ covered by sponges soaked in saline were used and held in place by a band. Stimulation was given at $2 \mathrm{~mA}$ for 20 min, with a gradual ramp up and ramp down of the current over $30 \mathrm{~s}$. For sham stimulation, a $1 \mathrm{~mA}$ current was applied for $30 \mathrm{~s}$ giving an initial sensation of tDCS while minimising stimulatory effects. Ramp up and ramp down was over $10 \mathrm{~s}$. The safety procedure utilised during stimulation was as previously described in Loo et al. ${ }^{14}$

\section{Assessment of mood and cognition}

The primary outcome measure for mood evaluation was the MADRS. Participants were evaluated at baseline, after sessions 8 , 15, 23 and 30, and at 1 week and 1 month after trial completion. Each participant was rated by the same psychiatrist or psychologist throughout the study using the MADRS, Inventory of Depressive Symptomatology (IDS) ${ }^{15}$ and Clinical Global Impression - Severity of Illness (CGI-S) ${ }^{16}$ scale. The clinician-rated Quick Inventory of Depressive Symptomatology (QIDS-C) ${ }^{17}$ score was also calculated as a subset of scores derived from the IDS. At the same time points, participants rated their mood using the self-rated version of the QIDS (QIDS-SR). ${ }^{17}$ At baseline, participants were also rated using the CORE Measure of Psychomotor Disturbance $(\mathrm{CORE})^{18}$ as a possible predictor of response.

Neuropsychological functioning was assessed at baseline, and after the sham-controlled (post-session 15) and open-label (post-session 30) phases using the following tests: Rey Auditory Verbal Learning Test (RAVLT), ${ }^{19}$ Digit Span Forwards and Backwards,${ }^{20}$ Stroop Test, ${ }^{21}$ Controlled Oral Word Association Test (COWAT) ${ }^{22}$ and Letter-Number Sequencing. ${ }^{23}$ Immediate effects of tDCS on processing speed were also assessed at treatment sessions 1 and 15 (tested immediately before and after stimulation) using the Symbol Digit Modalities Test (SDMT), ${ }^{24}$ and simple and choice reaction-time tests. Alternative test versions were used on different testing occasions for the RAVLT, COWAT and SDMT.

\section{Statistical analysis}

The two treatment groups were analysed for differences in demographic and clinical variables at baseline using $\chi^{2}$-tests for categorical variables and $t$-tests for continuous variables (Table 1). Statistical tests were two-tailed. Intention-to-treat lastobservation-carried-forward scores were used for the analyses below. Only those participants with at least one post-baseline rating were included in the analyses.

To test the effect of condition on mood over the course of treatment, for each depression rating, a $2 \times 2$ mixed betweenwithin ANCOVA covarying for baseline CORE scores was conducted with the between-groups factor being condition (active v. sham $\mathrm{tDCS}$ ) and the within-participants factor being time (baseline and post-session 15). Analyses tested for main effects of condition and time as well as the condition $\times$ time interaction. Baseline CORE ratings were correlated (Pearson's correlation) with the percentage change in MADRS scores over the 15 active treatment sessions (i.e. sessions 1-15 for active group, 16-30 for sham group) to examine whether CORE scores predicted response.

The number needed to treat (NNT) to obtain one responder to active treatment was calculated for active $v$. sham tDCS over the 3-week sham-controlled period. In a further exploratory 
analysis, the NNT to obtain a responder was also calculated for 6 weeks of active tDCS (i.e. tDCS received in the active group over the sham-controlled and open-label phases) v. 3 weeks of sham tDCS.

Scores from neuropsychological tests examining changes over the first 15 sessions (active or sham), and scores from tests administered immediately before and after sessions 1 and 15 (active or sham) were also analysed with a mixed between-within ANOVA testing for main effects of condition and time as well as condition $\times$ time interactions. In addition, neuropsychological tests scores were separately analysed for participants who received 30 active treatments (i.e. active group), examining for a main effect of time across the 30 sessions using a repeated measures ANCOVA controlling for the percentage change in MADRS scores over the same period.

To test the integrity of masking, a $\chi^{2}$-test was used to test for an association between participants' group allocation in the shamcontrolled phase and whether they guessed their treatment was 'active' or 'sham'.

\section{Results}

\section{Participants}

The only significant difference between the active and sham groups at baseline was higher CORE scores for the active group (Table 1). In total, 58 participants completed the 15-session sham-controlled phase with 52 participants going on to complete the open-label phase (Fig. 1). In total 13/31 participants (active group) and 10/29 participants (group initially assigned to sham treatment) received further sessions of tDCS (given weekly) during the 1-month follow-up period.

\section{Mood outcomes}

There were significant main effects of time over the shamcontrolled phase for all mood outcome measures indicating that scores were significantly lower at the end of the sham-controlled phase compared with baseline (Table 2). The only main effect of group was found in the QIDS-SR with higher scores for the sham group.

A significant interaction between group and time was found in MADRS scores (the primary outcome measure), with simple effects indicating that there was a greater decrease in scores from baseline to the end of the sham-controlled phase in the active group (Fig. 2) (effect size: 0.49). However, the same interaction only trended towards significance in QIDS-C scores and did not reach significance in the remaining mood outcome measures.

Over the sham-controlled phase, four participants in both the active and sham groups met the criterion for response. No participants in either group met the criterion for remission (MADRS score $<10$ ). By the end of the additional open-label phase, 15 participants in the active group and 12 participants in the sham group met the criterion for response. At 1-week follow-up the proportion of responders was 16/26 (active group) and 6/26 (sham group). At 1-month follow-up there were 13/20 (active group) and 7/23 (sham group) responders. The NNT was 16.7 for the 3-week active-sham comparison. The NNT for 6 weeks' active tDCS compared with 3 weeks' sham stimulation was 2.6. There was no significant correlation between CORE scores and percentage change in MADRS scores over the 3-week active treatment period.

\section{Neuropsychological functioning - sham $v$. active tDCS}

Over the sham-controlled phase, a significant main effect of time was found for Stroop Interference, indicating that participants were quicker in performing the task at the end of the shamcontrolled phase compared with baseline. However, there was no interaction between group and time (Table 3).

Analyses of participants' performance in the SDMT immediately before and after the first (tDCS 1) and final (tDCS 15) sessions in the sham-controlled phase, showed a significant effect of time only at the first tDCS session. In addition, there was a significant interaction between time and group with simple effects revealing that there was no difference between pre- and

\begin{tabular}{|c|c|c|c|c|c|c|c|c|c|c|}
\hline & \multicolumn{3}{|c|}{ Sham } & \multicolumn{3}{|c|}{ Active } & \multicolumn{3}{|c|}{ Test statistic } & \multirow[b]{2}{*}{$P$} \\
\hline & $n$ & Mean & s.d. & $n$ & Mean & s.d. & d.f. & $F$ & $\chi^{2}$ & \\
\hline \multicolumn{11}{|l|}{ Categorical variables } \\
\hline Gender, male/female & $15 / 14^{a}$ & & & $17 / 14^{a}$ & & & 1 & & 0.06 & 0.81 \\
\hline Melancholic/non-melancholic & $15 / 14^{\mathrm{a}}$ & & & $16 / 15^{\mathrm{a}}$ & & & 1 & & 0.00 & 0.99 \\
\hline Concurrent antidepressant medication, yes/no & $21 / 8^{\mathrm{a}}$ & & & $22 / 9^{a}$ & & & 1 & & 0.02 & 0.90 \\
\hline Bipolar diagnosis, yes/no & $4 / 25^{a}$ & & & $4 / 27^{a}$ & & & 1 & & 0.01 & 0.92 \\
\hline Edinburgh Handedness Inventory, right/left & $25 / 4^{\mathrm{a}}$ & & & $28 / 2^{a}$ & & & 1 & & 0.82 & 0.37 \\
\hline \multicolumn{11}{|l|}{ Continuous variables } \\
\hline Age, years & & 48.6 & 12.6 & & 47.8 & 12.5 & 1,58 & 0.23 & & 0.82 \\
\hline Age at onset, year & & 28.2 & 12.5 & & 28.3 & 12.6 & 1,58 & 0.25 & & 0.98 \\
\hline Duration of current episode, months & & 55.6 & 65 & & 33.8 & 56.2 & 1,58 & 1.39 & & 0.17 \\
\hline Duration of all previous episodes, months & & 82.3 & 93.2 & & 54.9 & 65.5 & 1,58 & 1.33 & & 0.19 \\
\hline Antidepressants failed current episode, $n$ : mean & & 1.79 & 2.14 & & 1.71 & 1.62 & 1,58 & 0.17 & & 0.87 \\
\hline Total lifetime failed antidepressants, $n$ : mean & & 2.79 & 3.36 & & 3.13 & 2.63 & 1,58 & 0.43 & & 0.67 \\
\hline Maudsley Staging parameters & & 6.93 & 2.51 & & 6.65 & 2.11 & 1,58 & 0.48 & & 0.63 \\
\hline Baseline MADRS score & & 29.5 & 4.96 & & 30.4 & 6.02 & 1,58 & 0.63 & & 0.53 \\
\hline Baseline IDS score & & 35.7 & 7.37 & & 36.6 & 9.63 & 1,57 & 0.41 & & 0.69 \\
\hline Baseline QIDS-C score & & 14.9 & 2.48 & & 15.3 & 3.50 & 1,58 & 0.59 & & 0.56 \\
\hline Baseline CGI score & & 4.28 & 0.53 & & 4.45 & 0.62 & 1,58 & 1.17 & & 0.25 \\
\hline Baseline CORE score & & 3.71 & 2.61 & & 6.40 & 5.51 & 1,42 & 2.40 & & 0.02 \\
\hline Baseline QIDS-SR score & & 16.0 & 3.26 & & 14.6 & 4.69 & 1,57 & 1.36 & & 0.18 \\
\hline
\end{tabular}

MADRS, Montgomery-Åsberg Depression Rating Scale; IDS, Inventory of Depressive Symptomatology; QIDS-C, Quick Inventory of Depressive Symptomatology (Clinician-rated): 

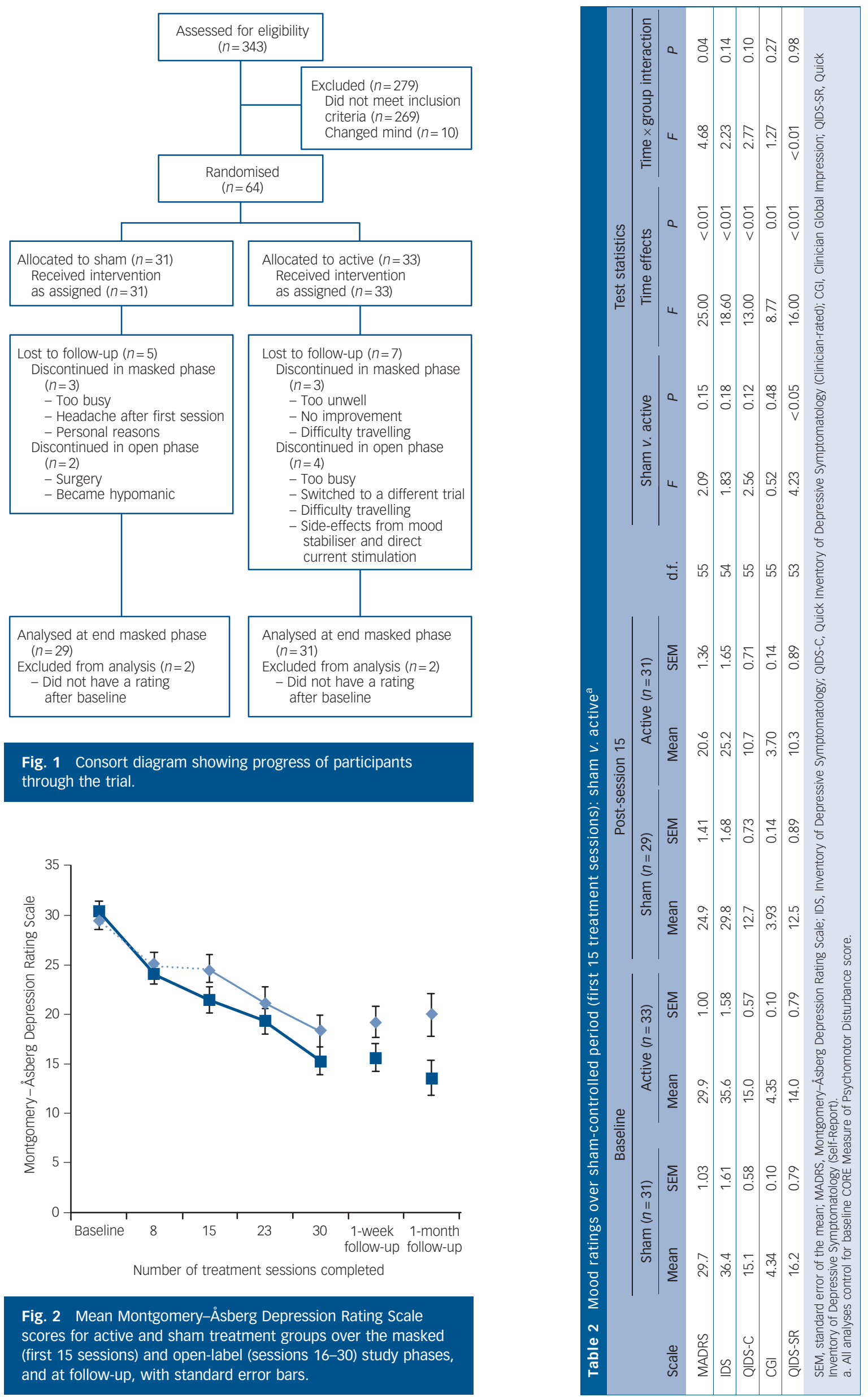

Fig. 1 Consort diagram showing progress of participants through the trial.

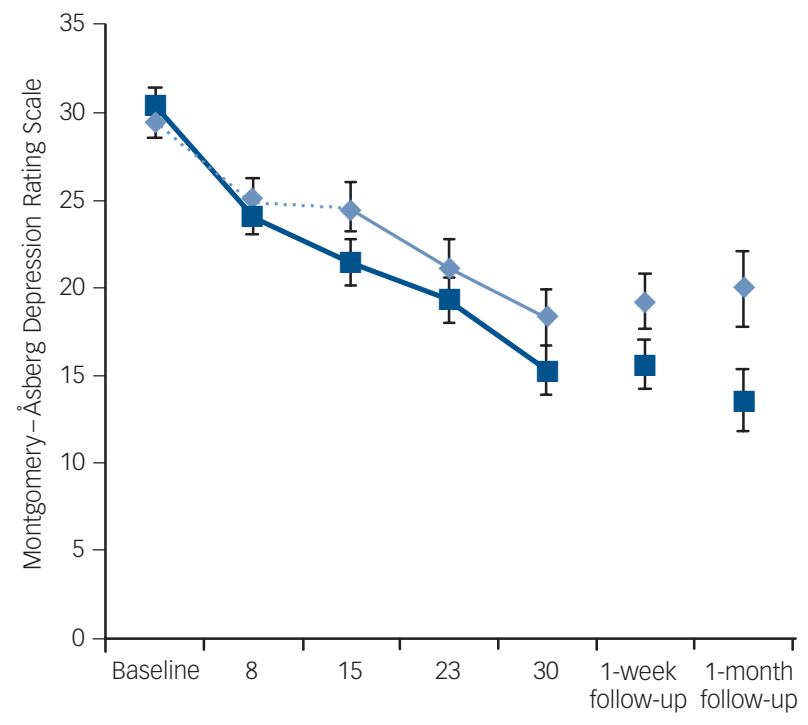

Number of treatment sessions completed

Fig. 2 Mean Montgomery-Åsberg Depression Rating Scale scores for active and sham treatment groups over the masked (first 15 sessions) and open-label (sessions 16-30) study phases, and at follow-up, with standard error bars. 

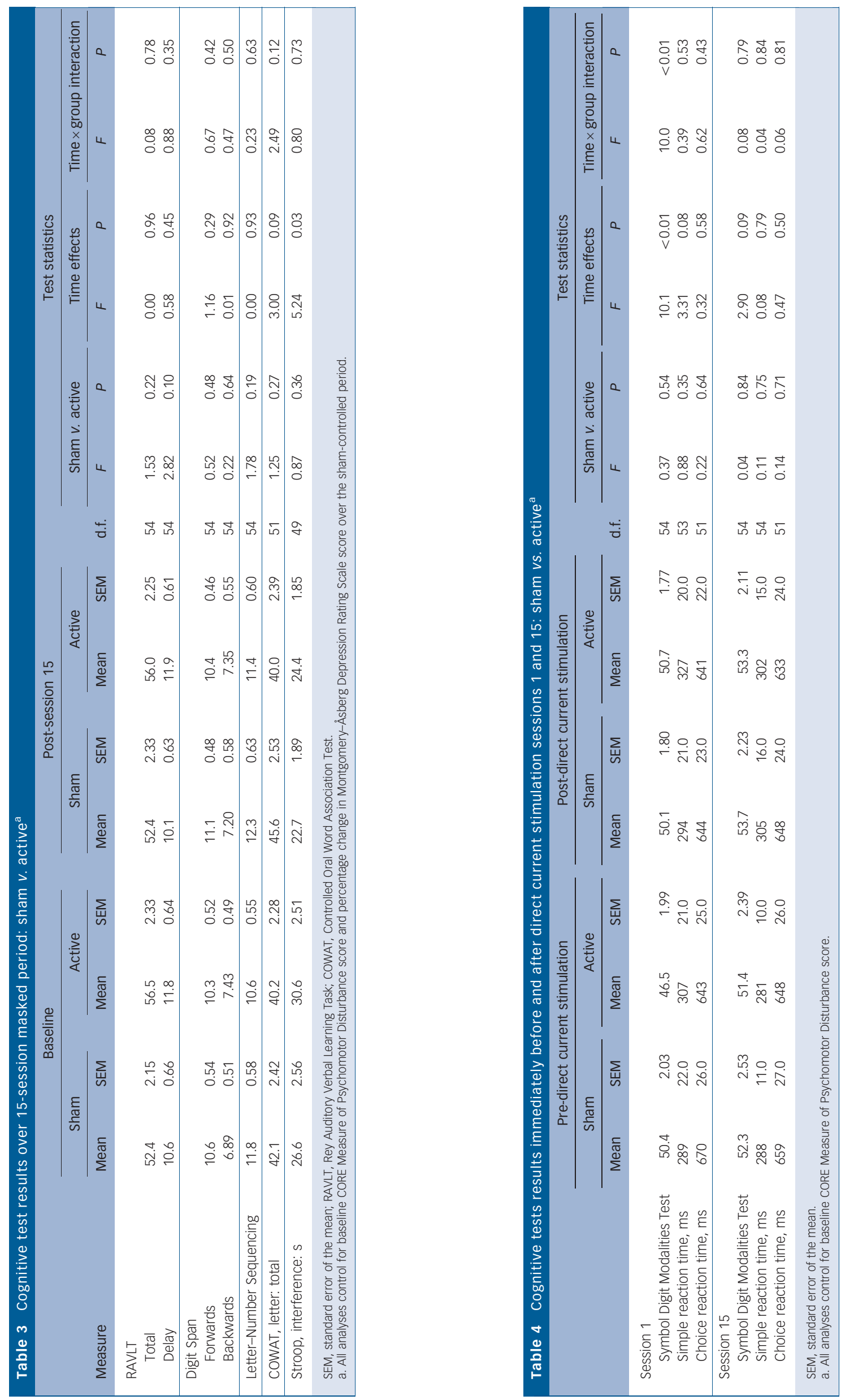
post-tDCS scores for the sham group, whereas scores in the active group significantly improved following tDCS (Table 4).

\section{Effects of 6 weeks of active tDCS on neuropsychological test performance}

In the active group (the only group to receive 6 weeks of active tDCS), there was a trend for main effect of time on the Letter-Number Sequencing Task, suggesting higher scores at 6 weeks compared with baseline $(F=3.46$, d.f. $=28, P=0.07)$. No significant differences over time were found within the other measures: RAVLT total $(F=0.12$, d.f. $=28, P=0.73)$, RAVLT delay $(F=0.41$, d.f. $=28, P=0.53)$, Digit Span Forwards $(F=1.02$, d.f. $=28, P=0.32)$, Digit Span Backwards $(F=0.01$, d.f. $=28$, $P=0.93)$, Stroop Interference $(F=0.24$, d.f. $=26, P=0.63)$ and COWAT $(F=1.72$, d.f. $=27, P=0.2)$.

\section{Adverse outcomes and side-effects}

During the sham-controlled phase, side-effects occurring during active treatment were skin redness (objectively observed) at the anode and/or cathode site $(n=30)$, tingling at anode and/or cathode site $(n=26)$, itching at anode and/or cathode site ( $n=23)$, burning/heating sensation at anode and/or cathode site $(n=14)$, headache $(n=12)$, dizziness/light-headedness $(n=10)$, fatigue $(n=7)$, nausea $(n=3)$, blurred vision $(n=3)$, pain at anode and/or cathode site $(n=2)$, pulsing sensation at anode and/or cathode site $(n=2)$, neck soreness $(n=1)$, visual effects when eyes closed $(n=1)$, seeing dots in the periphery $(n=1)$, giddiness $(n=1)$, flaky skin $(n=1)$, watery eyes $(n=1)$, a feeling of being 'spaced out' $(n=1)$ and shakiness $(n=1)$. In addition, one participant experienced transient hypomania in the open phase and was subsequently withdrawn from the trial. Side-effects occurring during sham treatment were skin redness at anode and/ or cathode site $(n=29)$, tingling at anode and/or cathode site $(n=27)$, itchiness at anode and/or cathode site $(n=22)$, headache ( $n=10)$, burning/heating sensation at anode and/or cathode site $(n=7)$, dizziness/light-headedness $(n=6)$, fatigue $(n=4)$, pulsing sensation at anode and/or cathode site $(n=2)$, right ear ache $(n=1)$, ringing in ears $(n=1)$, nausea $(n=1)$, twitching of right arm $(n=1)$, stiffness in neck and shoulders $(n=1)$, tingling on neck $(n=1)$, tingling on tongue $(n=1)$, a 'funny feeling' in head $(n=1)$, facial numbness $(n=1)$ and reflux $(n=1)$. All side-effects were transient and mostly ranged from mild to moderate in intensity.

\section{Integrity of masking}

When asked to guess their treatment assignment, correct responses were made by $14 / 24$ participants in the active group and $16 / 25$ participants in the sham group. The $\chi^{2}$-test indicated no significant difference between groups in the likelihood of active/sham guesses $\left(\chi^{2}=2.45\right.$, d.f. $\left.=1, P=0.12\right)$.

\section{Discussion}

\section{Efficacy}

This study reports the largest sample of individuals with current depression treated hitherto in a randomised controlled trial of tDCS. It used treatment parameters that exceeded those of previous trials, in an attempt to optimise efficacy. Overall, this study confirmed previous reports of significant antidepressant effects with tDCS, with the active treatment group showing significantly greater improvement compared with the sham group over the 3 -week study period on the primary outcome measure (change in MADRS). However, results over the 3 weeks were clinically modest, with only a $28 \%$ decline in MADRS scores after active stimulation, a small proportion of responders (which did not differ between active and sham treatment groups) and active-sham differences failing to reach significance on the other mood rating scales. The antidepressant effects measured in this study did not appear as robust as those previously reported in the sham-controlled trials of Fregni et al ${ }^{5}$ (60\% improvement after 5 sessions/ 1.5 weeks) and Boggio et al ${ }^{6}$ (43\% improvement after 10 sessions, 2 weeks), despite the use of tDCS at higher stimulus intensities, over a longer treatment period and with more stimulation sessions. These results in the active treatment group are comparable though, to those reported in our earlier trial of tDCS (37\% improvement after 10 sessions $/ 3.5$ weeks) ${ }^{7}$ and a recent open-label trial of tDCS (18\% improvement in unipolar depression after 10 sessions/ 1 week). ${ }^{25}$

The studies above did not differ obviously in sample characteristics but did differ in permitting concurrent antidepressant medication during tDCS. In the two trials with greater response, participants were free of medications prior to and during the trial (clarified in a personal communication from F. Fregni, March 2011), ${ }^{5,6}$ whereas in others ${ }^{7,25}$ and in this study, participants continued on antidepressant medication. Although participants in these latter trials were still significantly depressed at the time of commencing tDCS, the potential for further improvement with a physical treatment may have been limited compared with individuals not already receiving some treatment. A meta-analysis of trials of transcranial magnetic stimulation (TMS), another non-convulsive stimulatory treatment for depression, found greater efficacy when TMS was given as a monotherapy than when it was added to pre-existing pharmacotherapy. ${ }^{26}$

The trials above also differed in stimulus intensity and session frequency. Recent trials (Boggio et al, ${ }^{6}$ this trial, Brunoni et al ${ }^{25}$ ) increased both stimulus intensity $(2 \mathrm{~mA})$ and session frequency (daily or twice daily) in an attempt to optimise outcomes, however, results are not clearly superior to trials that used $1 \mathrm{~mA}$ and second daily treatments. ${ }^{5,7}$ Although the duration of stimulation has been shown to affect the duration of after effects, ${ }^{27}$ the influence of stimulus intensity and session frequency on efficacy are as yet unknown. In a recent study of tDCS to the motor cortex, we found that daily stimulation sessions led to greater cumulative changes in cortical excitability than second daily stimulation, over a given stimulation period (1 week), when all other parameters were kept constant. ${ }^{28}$ In a similar experiment, we did not find any difference in outcomes (cortical excitability) between gradual increase of stimulus intensity from $1 \mathrm{~mA}$ to $2 \mathrm{~mA}$ over five sessions conducted on consecutive weekdays, and maintaining stimulation intensity at $2 \mathrm{~mA}$ for all five session (details available from the authors on request). The effects of these variations in stimulus parameters on cortical effects warrant further systematic study.

Although results after 3 weeks of daily tDCS were modest, the number of responders after 6 weeks of treatment was much more encouraging, and comparable with outcomes from a recent large study of 6 weeks of TMS given on an open-label basis to participants with pharmacotherapy-resistant depression $(42.4 \%$ responders). ${ }^{29}$ The response rate was superior to that reported for antidepressant medication in individuals who had failed a first course of medication in the large Sequenced Treatment Alternatives to Relieve Depression $\left(\mathrm{STAR}^{\star} \mathrm{D}\right)$ study $(28.5 \%){ }^{30}$ This suggests that tDCS has meaningful antidepressant efficacy, including in those individuals resistant to pharmacotherapy. Comparison of MADRS outcomes and response rates between participants who received 3 and 6 weeks of active treatment 
suggest that further and more lasting benefit may be derived from extension of the treatment period to 6 weeks. Whether this is related to the number of treatments received (i.e. $30 v .15)$ or the overall duration of the treatment course ( 6 weeks rather than 3 weeks) cannot be determined from this study. Interpretation of these outcomes is limited by the fact that treatment in the second 3 weeks of the study was administered under open-label conditions.

\section{Cognitive effects}

Tests for acute cognitive effects following the first tDCS session showed improvement on the SDMT in the active tDCS group relative to the sham group. This finding suggests that tDCS may enhance acute attention and working memory in people with depression, in line with the acute attention-enhancing effects found following equivalent stimulation in individuals post-stroke. ${ }^{31}$

Consistent with our previous study, ${ }^{7}$ neuropsychological tests after 3 weeks ( 15 sessions) of active tDCS did not show any changes in performance across multiple cognitive domains. These results demonstrate that multiple tDCS sessions are safe and not associated with any adverse cognitive outcomes. Previously, Fregni et $a l^{32}$ showed that five daily tDCS sessions enhanced performance on the Digit Span Forwards and Backwards test, a finding we failed to replicate in a larger sample. ${ }^{7}$ Similarly, in the current study no improvement was found on these same measures after either 15 or 30 active tDCS sessions. This suggests that multiple tDCS sessions do not have cumulative cognitive enhancing effects independent of mood effects. Nonetheless, the use of more sensitive neurocognitive measures may help elucidate potential cognitive-enhancing effects of multiple tDCS treatments in future studies.

\section{Limitations and strengths}

A limitation of this study is that most participants were on antidepressant medications concurrently with tDCS. This was allowed in the study design for several reasons: ethical and scientific concerns that withdrawal of medications may have caused clinical deterioration prior to study entry; no evidence for safety issues with concurrent use of antidepressants and tDCS; and also because the continuation of medication while tDCS is introduced is likely to be the approach used if tDCS is eventually undertaken in clinical practice. Nevertheless, it means that the effects of tDCS could not be assessed independently of the presence of medications, which would be scientifically preferable. It is unlikely that the continuation of medications would have enhanced antidepressant effects, as these were medications to which participants had already failed to respond, and doses were stable for at least 4 weeks prior to study entry and during the study. On the contrary, this factor may have limited the scope of further antidepressant effects with tDCS, as discussed above.

Strengths of the study include the relatively large sample size, extension of tDCS stimulation to 30 sessions over 6 weeks, careful methodology with good participant adherence to the study protocol and detailed neuropsychological testing.

\section{Implications}

In conclusion, this trial studied tDCS at stimulation parameters beyond those previously used in depression and confirmed earlier reports of the antidepressant efficacy and safety of tDCS. The evidence to date suggests that tDCS is a potentially useful treatment for depression, and that treatment should be given for longer than 3 weeks for an adequate response.

\section{Funding}

This study was supported by an Australian National Health and Medical Research (NHMRC) council research grant number 510142

\section{Acknowledgements}

The authors thank Dusan Hadzi-Pavlovic for advice on statistical analysis, Michael Player, Jessica Cheung, Dr Aparna Menon, Vincent Chan, Joshua Garfield and Manasi Kogekar for assistance with data collection and processing, Professsor Walter Paulus and Dr Michael Nitsche for advice on tDCS, and Natalie Katalinic for assistance with manuscript preparation.

Colleen K. Loo, MB BS, MD, FRANZCP, School of Psychiatry, University of New South Wales, Sydney, St George Hospital, South Eastern Sydney Illawarra Health and Black Dog Institute, Sydney, Australia; Angelo Alonzo, BA, BSC (Hons), PhD, Donel Martin, MClinNeuroPsych, PhD, Philip B. Mitchell, AM, MB BS, MD, FRCPsych,

FRANZCP, School of Psychiatry, University of New South Wales and Black Dog

Institute, Sydney, Australia; Veronica Galvez, MD, School of Psychiatry, University of Institute, Sydney, Australia; Veronica Galvez, MD, School of Psychiatry, University of
New South Wales, Sydney, Black Dog Institute, Sydney, and Mood Disorders Clinical and Research Unit, Psychiatry Department, Bellvitge University Hospital \& Neuroscience Group, IDIBELL (Bellvitge Biomedical Research Institute) L'Hospitalet de Llobregat, Barcelona, Spain; Perminder Sachdev, MB BS, MD, PhD, FRANZCP, School of Psychiatry, University of New South Wales and Neuropsychiatric Institute, Prince of Wales Hospital, Randwick, Sydney, Australia

Correspondence: Colleen Loo, Level 2, James Laws House, St George Hospital, Kogarah, NSW 2217, Australia. Email: colleen.loo@unsw.edu.au

First received 6 Jun 2011, final revision 9 Oct 2011, accepted 26 Oct 2011

\section{References}

1 Nitsche MA, Cohen LG, Wassermann EM, Priori A, Lang N, Antal A, et al Transcranial direct current stimulation: state of the art 2008. Brain Stimul 2008; 1: 206-23.

2 Bindman L, Lippold OC, Redfearn JW. The action of brief polarizing currents on the cerebral cortex of the rat (1) during current flow and (2) in the production of long-lasting after-effects. J Physiol 1964; 172: 369-82.

3 Arul-Anandam AP, Loo C. Transcranial direct current stimulation: a new tool for the treatment of depression? J Affect Disord 2009; 117: 137-45.

4 Nitsche MA, Boggio PS, Fregni F, Pascual-Leone A. Treatment of depression with transcranial direct current stimulation (tDCS): a review. Exp Neurol 2009; 219: 14-9.

5 Fregni F, Boggio PS, Nitsche MA, Marcolin MA, Rigonatti SP, Pascual-Leone A. Treatment of major depression with transcranial direct current stimulation. Bipolar Disord 2006; 8: 203-4.

6 Boggio PS, Rigonatti SP, Ribeiro RB, Myczkowski ML, Nitsche MA Pascual-Leone A, et al. A randomized, double-blind clinical trial on the efficacy of cortical direct current stimulation for the treatment of major depression. Int J Neuropsychopharmacol 2008; 11: 249-54.

7 Loo CK, Sachdev P, Martin D, Pigot M, Alonzo A, Malhi GS, et al. A doubleblind, sham-controlled trial of transcranial direct current stimulation for the treatment of depression. Int J Neuropsychopharmacol 2010; 13: 61-9.

8 American Psychiatric Association. Diagnostic and Statistical Manual of Mental Disorders (4th edn) (DSM-IV). APA, 1994.

9 Montgomery SA, Åsberg M. A new depression scale designed to be sensitive to change. Br J Psychiatry 1979; 134: 382-9.

10 Sheehan DV, Lecrubier $\mathrm{Y}$, Sheehan $\mathrm{KH}$, Amorim $\mathrm{P}$, Janavs J, Weiller $\mathrm{E}$, et al. The Mini-International Neuropsychiatric Interview (M.I.N.I): the development and validation of a structured diagnostic psychiatric interview for DSM-IV and ICD-10. J Clin Psychiatry 1998; 59 (suppl 20): 22-33.

11 Liebetanz D, Nitsche MA, Tergau F, Paulus W. Pharmacological approach to the mechanisms of transcranial DC-stimulation-induced after-effects of human motor cortex excitability. Brain 2002; 125: 2238-47.

12 Nitsche MA, Liebetanz D, Schlitterlau A, Henschke U, Fricke K, Frommann K, et al. GABAergic modulation of DC stimulation-induced motor cortex excitability shifts in humans. Eur J Neurosci 2004; 19: 2720-6.

13 Fekadu A, Wooderson SC, Markopoulou K, Cleare AJ. The Maudsley Staging Method for treatment-resistant depression: prediction of longer-term outcome and persistence of symptoms. J Clin Psychiatry 2009; 70: 952-7.

14 Loo CK, Martin DM, Alonzo A, Gandevia S, Mitchell PB, Sachdev P. Avoiding skin burns with transcranial direct current stimulation: preliminary considerations. Int J Neuropsychopharmacol 2011; 14: 1-2. 
15 Rush AJ, Gullion CM, Basco MR, Jarrett RB, Trivedi MH. The Inventory of Depressive Symptomatology (IDS): psychometric properties. Psychol Med 1996; 26: 477-86.

16 Guy W. Clinical global impressions. In Assessment Manual for Psychopharmacology - Revised: 218-22. National Insitute of Mental Health 1976.

17 Rush AJ, Trivedi MH, Ibrahim HM, Carmody TJ, Arnow B, Klein DN, et al. The 16-Item Quick Inventory of Depressive Symptomatology (QIDS), clinician rating (QIDS-C), and self-report (QIDS-SR): a psychometric evaluation in patients with chronic major depression. Biol Psychiatry 2003; 54: 573-83.

18 Parker G, Hadzi-Pavlovic D, Boyce P, Wilhelm K, Brodaty H, Mitchell P, et al Classifying depression by mental state signs. Br J Psychiatry 1990; 157: 55-65.

19 Rey A. L'Examen Clinique en Psychologie [in French]. Presses Universitaires de France, 1964

20 Wechsler D. WAIS-R Manual. Psychological Corporation, 1981.

21 Spreen O, Strauss E. A Compendium of Neuropsychological Tests (2nd edn). Oxford University Press, 1998.

22 Benton AL, Hamsher KD. Multilingual Aphasia Examination. AJA Associates, 1989.

23 Wechsler D. Wechsler Adult Intelligence Scale - Administration and Scoring Manual (3rd edn). The Psychological Corporation, 1997.

24 Smith A. Symbol Digit Modalities Test. Western Psychological Services, 1991

25 Brunoni AR, Ferrucci R, Bortolomasi M, Vergari M, Tadini L, Boggio PS, et al. Transcranial direct current stimulation (tDCS) in unipolar vs, bipolar depressive disorder. Prog Neuropsychopharmacol Biol Psychiatry 2011; 35: 96-101.

26 Slotema CW, Blom JD, Hoek HW, Sommer IE. Should we expand the toolbox of psychiatric treatment methods to include repetitive transcranial magnetic stimulation (rTMS)? A meta-analysis of the efficacy of rTMS in psychiatric disorders. J Clin Psychiatry 2010; 71: 873-84.

27 Nitsche MA, Paulus W. Sustained excitability elevations induced by transcranial DC motor cortex stimulation in humans. Neurology 2001; 57: 1899-901.

28 Alonzo A, Brassil J, Taylor J, Martin D, Loo CK. Daily transcranial direct current stimulation (tDCS) leads to greater increases in cortical excitability than second daily tDCS. Brain Stimul 2011; May 25 (Epub ahead of print).

29 Avery DH, Isenberg KE, Sampson SM, Janicak PG, Lisanby SH, Maixner DF, et al. Transcranial magnetic stimulation in the acute treatment of major depressive disorder: clinical response in an open-label extension trial. J Clin Psychiatry 2008; 69: 441-51.

30 Rush AJ, Trivedi MH, Wisniewski SR, Nierenberg AA, Stewart JW, Warden D, et al. Acute and longer-term outcomes in depressed outpatients requiring one or several treatment steps: a STAR*D report. Am J Psychiatry 2006; 163 1905-17.

31 Kang EK, Baek MJ, Kim S, Paik NJ. Non-invasive cortical stimulation improves post-stroke attention decline. Restor Neurol Neurosci 2009; 27: 645-50.

32 Fregni F, Boggio PS, Nitsche MA, Rigonatti SP, Pascual-Leone A. Cognitive effects of repeated sessions of transcranial direct current stimulation in patients with depression. Depress Anxiety 2006; 23: 482-4.

\section{extra}

\section{The butterfly}

\section{Shabbir Amanullah}

As adults, we consider ourselves capable of interpreting, at least to some extent, the behaviour of children. Many a text has been written about childhood behaviour and its interpretation. We use strategies to reinforce or root out certain behaviours. However, we often fail to grasp the immense strength of a child's imagination. On Canada Day we were at a park, waiting for the fireworks display to begin at sundown. It was a warm day and children were running around the place. There was the usual popcorn, fizzy drinks, ice cream, lobster (exclusive to Prince Edward Island), balloons and, of course, face painting. While sitting beside a fence with a few friends, we saw a child coming towards us. She was beaming and seemed extremely content. She had a butterfly painted on her face. It was in three different colours and covered her face entirely. Thinking that she had come to show us all that she 'acquired' at the fair so far, we commented on the different things she had with her. In a typically childish way, she explained how she got them. Then, as she was about to leave, I said, 'You have a pretty butterfly painted on your face'. She looked at me in stunned silence and then replied in a huff 'I am the butterfly! We fail to comprehend the extent, depth and importance of the child's imagination by assuming we 'know'. 